\title{
Composição corporal e expansibilidade toracoabdominal em idosas praticantes de programa exercício físico supervisionado
}

\author{
Body composition and thoracoabdominal expansion in elderly women \\ submitted to a supervised physical exercise program
}

\author{
Tábata de Paula Facioli ${ }^{\natural} \bowtie$, Regina Celi Trindade Camargo ${ }^{2}$, Elaine Aparecida Lozano da Silva ${ }^{2}$, \\ Juliana Rosini da Silva², Cláudia Regina Sgobbi de Faria ${ }^{2}$, Mariana Carvalho Pinto ${ }^{2}$ \\ 1 Faculdade de Medicina de Ribeirão Preto da Universidade de São Paulo (USP). Ribeirão Preto, SP, Brasil. \\ ${ }^{2}$ Faculdade de Ciências e Tecnologia (FCT) da Universidade Estadual Paulista Júlio de Mesquita Filho (UNESP). Presidente Prudente, SP, Brasil.
}

\section{RESUMO}

Objetivos: Avaliar os efeitos do exercício físico supervisionado sobre a composição corporal e a expansibilidade toracoabdominal de mulheres idosas inseridas em um programa de exercício físico regular e supervisionado.

Métodos: Foram incluídas mulheres com idade igual ou superior a 60 anos, integrantes de um programa de exercício físico supervisionado, com frequência superior a $75 \%$. As participantes foram dividas em dois grupos: programa duas vezes por semana (P2) e programa três vezes por semana (P3), sendo avaliadas antes do início do programa e após seis meses. Para as medidas de circunferência durante os movimentos respiratórios, foi realizada a cirtometria nas regiões axilar, xifoide, $12^{\text {as }}$ costelas e região umbilical; e para a avaliação da composição corporal, a bioimpedância.

Resultados: A amostra constou de 60 idosas, 29 pertencentes ao P2, com média de idade de 73,93 $\pm 6,76$ anos, e 31 pertencentes ao P3, com média de idade de $67,41 \pm 5,61$ anos. A cirtometria demonstrou que em P2 houve aumento do coeficiente respiratório das $12^{\text {as }}$ costelas entre o início (média 1,94 $\pm 1,67 \mathrm{~cm}$ ) e o fim do programa (média 3,07 $\pm 1,67 \mathrm{~cm}$ ), p=0,018; e em P3 houve aumento do coeficiente respiratório na região axilar entre a avaliação inicial (média $2,17 \pm 0,97 \mathrm{~cm}$ ) e a final (média 2,84 $\pm 1,81 \mathrm{~cm}$ ), p=0,030. Nas outras variáveis em estudo não houve modificações. Na comparação entre os grupos, não houve diferenças significativas entre as medidas correspondentes a cada período da avaliação. Com relação à composição corporal, o grupo P2 apresentou aumento em massa magra e diminuição de gordura corporal entre o início e o fim do programa, enquanto as participantes do P3 mantiveram os mesmos valores.

Conclusões: Após seis meses de participação em um programa de exercício físico supervisionado com sessões duas ou três vezes por semana, mulheres idosas demonstraram alguma melhora na expansibilidade toracoabdominal, aumento da massa magra e diminuição e/ou manutenção da gordura corporal.

DESCRITORES: exercício físico; composição corporal; saúde do idoso.

\section{ABSTRACT}

Aims: To evaluate the effects of supervised exercise on body composition and thoracoabdominal expansion of women submitted to a regular and supervised exercise program.

Methods: Women aged 60 years or older participating in a supervised exercise program, with attendance greater than $75 \%$, were included in the study. The subjects were divided into two groups: exercises twice a week (P2) and three times a week (P3), evaluated before the program and after six months. The circumference measurements during respiratory motion were obtained in the axillary, xiphoid, and umbilical regions, and in the $12^{\text {th }}$ pair of ribs, whereas body composition was assessed by bioimpedance.

Results: The sample consisted of 60 elderly women, 29 of whom belonged to P2, with a mean age of $73.93 \pm 6.76$ years, and 31 to P3, with a mean age of $67.41 \pm 5.61$ years. Cirtometry demonstrated a higher respiratory coefficient for the $12^{\text {th }}$ pair of ribs in P2 between the beginning (mean $1.94 \pm 1.67 \mathrm{~cm}$ ) and end of the program (mean of $3.07 \pm 1.67 \mathrm{~cm}$ ), p=0.018, and a higher respiratory coefficient in the axillary region in $\mathrm{P} 3$ at baseline (mean of $2.17 \pm 0.97 \mathrm{~cm}$ ) and at the end of the program (mean of $2.84 \pm 1.81 \mathrm{~cm}$ ), $\mathrm{p}=0.030$. The other variables remained unchanged. No significant differences were observed when the groups were compared. Regarding body composition, the P2 group had an increase in lean body mass and a decrease in body fat between the beginning and end of the program, while the P3 group maintained the same indices. Conclusions: The elderly women revealed some improvement in thoracoabdominal expansion, increase in lean body mass, and a decrease or maintenance of body fat after six months in the supervised exercise program, with sessions twice or three times a week.

KEY WORDS: exercise, physical; body composition; health of the elderly.

Recebido: maio de 2015 
Abreviaturas: IMC, índice de massa corporal; EFS, exercício físico supervisionado; MM, massa magra; GC, gordura corporal; CR, coeficiente respiratório; RPG, reeducação postural global; P2, grupo com exercício físico supervisionado duas vezes por semana; P3, grupo com exercício físico supervisionado três vezes por semana.

\section{INTRODUÇÃO}

O envelhecimento é um processo múltiplo e complexo que tem sido foco de atenção crescente em todo o mundo. O número de pessoas que chega à chamada terceira idade está aumentando gradativamente. Aspectos relativos à qualidade de vida dessa população tornam-se objetos de preocupação e de estudos, pois se referem a um fenômeno fisiológico de comportamento social ou cronológico de contínuas mudanças no domínio biopsicossocial ao longo da vida [1]. A faixa etária de 60 anos ou mais tende a aumentar em termos absolutos, no período de 2000 a 2050, passando de 600 mil para dois bilhões em 2050, provavelmente representando nesse ano $75 \%$ do total da população [2].

Com a constatação do significativo aumento da expectativa média de vida, a preocupação da sociedade acentua-se em promover maior grau de satisfação e menor grau de perdas ao idoso, sendo fundamentais a implantação de programas específicos para a promoção da saúde e a prevenção de doenças relacionadas ao envelhecimento [3]. Baltes [4], em 1987, já se referiu a este conceito como o idealizado envelhecimento bem sucedido $[3,4]$.

Deve-se salientar que o envelhecimento é considerado um processo influenciável pelo avançar da idade, sofrendo perdas inevitáveis pelos declínios biológicos normais dessa etapa da vida, mas que não são inteiramente responsáveis pelas doenças e dependência física na velhice, já que o desuso do corpo pode acentuar ainda mais essas perdas [5]. A inatividade física (sedentarismo) implica em custo econômico para o indivíduo, a família e a sociedade. O Centers for Disease Control and Prevention, nos Estados Unidos, aponta o sedentarismo como responsável por mais de dois milhões de mortes por ano, em função da sua repercussão no incremento de doenças crônicas não transmissíveis. Unindo-se a estes fatos, outro fenômeno cada vez mais expressivo é o aumento da obesidade da população, decorrente de hábitos alimentares inadequados [6,7].

A prática de exercício físico deve ser estimulada não somente para o idoso, mas para pessoas adultas em geral, sendo o instrumento fundamental para prevenção e controle das doenças crônicas não transmissíveis advindas do processo degenerativo do envelhecimento, assim como forma de manter a independência funcional e auxiliar na redução de estressores psicossociais sobre o indivíduo. Adotar essas mudanças é fundamental para um envelhecimento saudável $[8,9]$.

Benefícios específicos podem ser alcançados, dentre eles o ganho de massa magra e a redução de gordura corpórea, o que deve ser constantemente buscado, já que a obesidade está presente hoje em todas as faixas etárias e classes sociais. Exercícios aeróbios e anaeróbicos são benéficos para perda de peso, podendo seus efeitos ser potencializados pela associação de uma dieta hipocalórica e exercícios com pesos, por levarem ao aumento de massa magra [10].

Um instrumento bastante utilizado para avaliação da composição corporal é a bioimpedância elétrica, a qual utiliza uma corrente elétrica de baixa intensidade. Considerada uma técnica segura, rápida, de baixo custo e de grande aplicabilidade na prática clínica e em estudos, a bioimpedância estima a água corporal total, a massa magra e a quantidade de gordura corporal, sendo um método utilizado em crianças, adultos e idosos [11-13].

Dentre todos os sistemas que compõem o corpo humano, o respiratório pode ser considerado o sistema do organismo que envelhece mais rapidamente, por sofrer grande exposição a poluentes ao longo da vida. Alterações corporais como peso e estatura, atribuídas ao envelhecimento, podem também provocar alterações pulmonares, e, portanto, é valido o seu estudo detalhado [14]. Diminuição da estatura, substituição de músculo por tecido adiposo, alterações no perímetro abdominal e no índice de massa corporal (IMC) têm influência relevante no desempenho respiratório $[15,16]$.

Estudos epidemiológicos indicam que a prática de exercício físico regular pode melhorar a qualidade de vida e promover a saúde física e mental dos idosos. Para isso, eles devem realizar atividades que exijam atenção, concentração, coordenação e pensamento lógico $[7,17]$. Partindo-se desses preceitos, o presente estudo teve como objetivo avaliar alterações na composição corporal e na expansibilidade torácica de mulheres com idade igual ou superior a 60 anos, participantes de programa de fisioterapia gerontológica preventiva, com enfoque em exercício físico supervisionado (EFS) regular.

\section{MÉTODOS}

Este estudo foi realizado no período de junho a dezembro de 2012, com participantes de um programa 
de fisioterapia com EFS para a terceira idade no setor de gerontologia do Centro de Estudos e Atendimentos em Fisioterapia e Reabilitação (CEAFIR) e no Núcleo Morumbi da Faculdade de Ciências e Tecnologia (FCT), ambos da Universidade Estadual Paulista Júlio de Mesquita Filho (UNESP). O estudo foi aprovado pelo Comitê de Ética em Pesquisa da UNESP conforme parecer consubstanciado de número 51910.

Foram incluídas no estudo mulheres com idade igual ou superior a 60 anos, participantes do programa, com estabilidade clínica, com frequência superior a $75 \%$ nas aulas e que aceitaram a proposta de avaliação. Foram excluídas cardiopatas, pneumopatas, pacientes com realização recente de algum procedimento cirúrgico ou com algum outro tipo de intercorrência clínica no decorrer das avaliações.

As avaliações foram realizadas nos locais onde ocorriam as atividades do programa. As participantes foram agrupadas de acordo com o programa em que estavam vinculadas: atividades duas vezes por semana (P2) ou atividades três vezes por semana (P3). Na avaliação inicial foram coletados os dados pessoais de cada participante, e realizadas a cirtometria (conjunto de medidas das circunferências de tórax e abdômen durante os movimentos respiratórios) e a bioimpedância para avaliação da composição corporal.

Para a realização da cirtometria foi utilizada uma fita métrica com escala de 0 a 150 centímetros. A participante permaneceu em posição ortostática e o examinador à frente da mesma. As medidas foram realizadas em quatro regiões do tórax: (1) perímetro axilar com fita métrica passando pelos cavos axilares ao nível da terceira costela; (2) perímetro xifóide, passando sobre o apêndice xifóide ao nível da sétima cartilagem costal; (3) perímetro basal, passando sobre as $12^{\text {as }}$ costelas; e (4) perímetro umbilical, passando sobre a cicatriz umbilical [15]. Foi feita a mensuração em cada uma dessas quatro regiões, primeiramente durante a inspiração máxima (ao nível da capacidade pulmonar total) e, posteriormente, na expiração máxima (ao nível do volume residual) [18].

A análise da composição corporal foi realizada com equipamento de bioimpedância tetrapolar da marca Maltron modelo BF-906 (Maltron International Ltd., Rayleigh, Inglaterra). Foram colocados quatro eletrodos no hemicorpo direito da idosa, sendo dois no membro superior e dois no membro inferior. No membro superior um eletrodo foi colocado na articulação metacarpo-falangeana do terceiro dedo e o outro foi fixado pouco acima da articulação do punho, entre as proeminências distais de rádio e ulna, próximo ao processo estiloide. No membro inferior um eletrodo foi colocado na superfície dorsal do pé, na região metatarso-falangeana do terceiro artelho, e o outro um pouco acima da articulação do tornozelo, medialmente entre os maléolos medial e lateral [19]. Os dados obtidos após leitura do equipamento de bioimpedância foram quantidade de massa magra (MM), gordura corporal (GC), peso ideal, metabolismo energético basal, água corporal total e ideal, bem como o IMC predito.

Durante todo o período do estudo as participantes foram questionadas sobre restrição ou acompanhamento alimentar e sobre as demais atividades realizadas. No início e ao final do período de treinamento, todas as mulheres responderam verbalmente às seguintes perguntas: "Você realiza/realizou qualquer outro tipo de exercício físico durante o período de exercício físico supervisionado?" "Você realiza/realizou alguma restrição/acompanhamento nutricional por conta própria ou com a ajuda de um profissional nutricionista durante o período de exercício físico supervisionado?" As respostas foram anotadas nas fichas de avaliação $\mathrm{e}$ as participantes foram orientadas a informar aos pesquisadores qualquer alteração de suas respostas durante o decorrer do estudo.

Depois de efetuadas as avaliações iniciais, durante um período de seis meses as participantes realizaram um protocolo de EFS e mantiveram as suas atividades diárias normalmente. O protocolo de EFS foi composto por alongamentos globais, aquecimento contendo atividades que envolveram flexibilidade, equilíbrio e coordenação, (aproximadamente 15 minutos); exercícios de fortalecimento muscular de membros superiores e inferiores com utilização de pesos livres e halteres que variaram entre $0,5 \mathrm{~kg}$ e $2 \mathrm{~kg}$, bem como bandas elásticas; conscientização corporal e trabalho postural (20 minutos); um momento para a realização de jogos lúdicos a fim de exercitar a memória e a socialização (10 minutos); e ao final, um período de relaxamento/alongamento, englobando exercícios respiratórios (5 minutos). No início e no término de cada sessão foram aferidos sinais vitais, pressão arterial e frequência cardíaca. Após o período de seis meses de EFS foram repetidas todas as avaliações realizadas inicialmente.

A estatística descritiva foi composta por valores de média e desvio-padrão. As comparações entre o momento inicial e final foram efetuadas pelo teste $t \mathrm{de}$ Student para dados pareados, no qual se adotou valores de significância estatística inferiores a $5 \%(p<0,05)$. Todas as análises foram efetuadas no software BioEstat versão 5.0 . 


\section{RESULTADOS}

A amostra constou de 60 idosas, sendo 29 pertencentes ao $\mathrm{P} 2$, com média de idade de $73,93 \pm 6,76$ anos, e 31 mulheres pertencentes ao P3, com média de

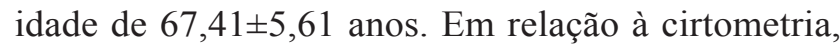
as médias da mobilidade torácica das quatro regiões mensuradas, para o P2 e P3, são descritas na Tabela 1. Observa-se que no $\mathrm{P} 2$ houve aumento do coeficiente respiratório (CR) das $12^{\text {as }}$ costelas e no $\mathrm{P} 3$ houve aumento do $\mathrm{CR}$ da região axilar, entre o início e o fim do programa. Nas outras variáveis em estudo não houve modificações.

A análise do IMC, percentil de MM e GC de ambos os grupos são vistos na Tabela 2. Observa-se que em ambos os grupos as médias de IMC encontravamse entre 25 e $29,99 \mathrm{~kg} / \mathrm{m}^{2}$, e somente P2 apresentou melhora na composição corporal, com diminuição de GC e aumento de MM, enquanto que P3 manteve seus valores.

Em relação à realização ou não de outro tipo de exercício físico e aos aspectos nutricionais,
14 (48,27\%) participantes do P2 relataram ter praticado exercícios físicos fora do programa, enquanto apenas três $(10,0 \%)$ participantes do P3 o fizeram. Cinco $(17,24 \%)$ participantes do $\mathrm{P} 2$ e cinco $(16,66 \%)$ do P3 relataram ter tido alguma restrição/ acompanhamento nutricional durante o período do programa de EFS (diferença não significativa).

\section{DISCUSSÃO}

No presente estudo foi realizada a mensuração da mobilidade torácica em 60 idosas participantes de um programa de EFS por meio da cirtometria, utilizando quatro pontos de mensuração, assim como avaliada a composição corporal pela bioimpedância. Os resultados demonstraram que P2 (grupo que realizava sessões de exercício duas vezes por semana) obteve melhores respostas ao programa de EFS proposto em relação a P3 (que realizava as sessões três vezes por semana), já que apresentou resultados positivos em três quesitos, $\mathrm{CR}$ das $12^{\text {as }}$ costelas, MM e GC, enquanto P3 somente obteve melhora na $\mathrm{CR}$ da região axilar.

Tabela 1. Resultados da cirtometria de 60 mulheres idosas participantes de um programa de exercício físico supervisionado, com sessões duas vezes por semana (P2) ou três vezes por semana (P3), durante seis meses. Centro de Estudos e Atendimentos em Fisioterapia e Reabilitação (CEAFIR) e no Núcleo Morumbi da Faculdade de Ciências e Tecnologia (FCT), junho a dezembro de 2012.

\begin{tabular}{|c|c|c|c|c|c|c|}
\hline \multirow{2}{*}{ Medidas $(\mathrm{cm})$} & \multicolumn{3}{|c|}{$\begin{array}{l}\text { P2 (duas vezes por semana) } \\
\qquad \mathrm{N}=29\end{array}$} & \multicolumn{3}{|c|}{$\begin{array}{l}\text { P3 (três vezes por semana) } \\
\qquad \mathrm{N}=31\end{array}$} \\
\hline & $\begin{array}{l}\text { Avaliação inicial } \\
\text { (média } \pm \text { DP) }\end{array}$ & $\begin{array}{l}\text { Avaliação final } \\
\text { (média } \pm \mathrm{DP})\end{array}$ & $\mathbf{p}^{*}$ & $\begin{array}{l}\text { Avaliação inicial } \\
\text { (média } \pm \text { DP) }\end{array}$ & $\begin{array}{l}\text { Avaliação final } \\
\text { (média } \pm \text { DP) }\end{array}$ & $\mathbf{p}^{*}$ \\
\hline CR axilar & $2,34 \pm 1,57$ & $3,13 \pm 1,70$ & 0,089 & $2,17 \pm 0,97$ & $2,84 \pm 1,81$ & 0,030 \\
\hline CR xifóide & $2,26 \pm 2,37$ & $2 ., 90 \pm 1,67$ & 0,271 & $1,75 \pm 2,03$ & $2,67 \pm 1,97$ & 0,104 \\
\hline CR $12^{\text {as }}$ costelas & $1,94 \pm 1,67$ & $3,07 \pm 1,67$ & 0,018 & $1,65 \pm 1,30$ & $2,25 \pm 1,74$ & 0,170 \\
\hline CR umbilical & $1,92 \pm 2,41$ & $2,65 \pm 1,26$ & 0,178 & $1,38 \pm 0,99$ & $1,67 \pm 1,40$ & 0,397 \\
\hline
\end{tabular}

DP: desvio padrão; CR: coeficiente respiratório.

* Teste $t$ de Student para dados pareados.

Tabela 2. Resultados da composição corporal obtidos por bioimpedância em 60 mulheres idosas participantes de um programa de exercício físico supervisionado, com sessões duas vezes por semana (P2) ou três vezes por semana (P3), durante seis meses. Centro de Estudos e Atendimentos em Fisioterapia e Reabilitação (CEAFIR) e no Núcleo Morumbi da Faculdade de Ciências e Tecnologia (FCT), junho a dezembro de 2012.

\begin{tabular}{|c|c|c|c|c|c|c|}
\hline \multirow{2}{*}{ Medidas $\left(\mathrm{kg} / \mathrm{m}^{2}\right)$} & \multicolumn{3}{|c|}{$\begin{array}{l}\text { P2 (duas vezes por semana) } \\
\qquad N=29\end{array}$} & \multicolumn{3}{|c|}{$\begin{array}{c}\text { P3 (três vezes por semana) } \\
\qquad N=31\end{array}$} \\
\hline & $\begin{array}{l}\text { Avaliação inicial } \\
\text { (média } \pm \mathrm{DP})\end{array}$ & $\begin{array}{l}\text { Avaliação final } \\
\text { (média } \pm \text { DP) }\end{array}$ & $\mathbf{p}^{*}$ & $\begin{array}{l}\text { Avaliação inicial } \\
\text { (média } \pm \mathrm{DP})\end{array}$ & $\begin{array}{l}\text { Avaliação inicial } \\
\text { (média } \pm \text { DP) }\end{array}$ & $\mathbf{p}^{*}$ \\
\hline IMC & $26,50 \pm 3,925$ & $26,23 \pm 4,076$ & 0,069 & $28,41 \pm 4,664$ & $28,66 \pm 4,576$ & 0,190 \\
\hline GC & $39,63 \pm 6,532$ & $32,26 \pm 7,811$ & $<0,001$ & $42,54 \pm 5,762$ & $41,36 \pm 6,882$ & 0,198 \\
\hline MM & $60,33 \pm 6,585$ & $67,73 \pm 7,791$ & $<0,001$ & $57,49 \pm 5,687$ & $58,61 \pm 6,767$ & 0,206 \\
\hline
\end{tabular}

DP: desvio padrão; IMC: índice de massa corporal; GC: gordura corporal; MM: massa magra.

* Teste t de Student para dados pareados. 
Poucos estudos enfocaram a técnica da cirtometria como forma de avaliação da mobilidade torácica, por esse motivo não há padronização de como a mesma deve ser realizada, ou seja, quais as regiões que devem ser mensuradas e os valores que devem ser considerados. Encontram-se na literatura estudos relatando que a técnica deveria ser realizada mensurando quatro regiões, porém estas diferem entre axilar, mamilar, xifoideana e basal [20] e axilar, xifoideana, basal e umbilical [21], sendo ainda possível encontrar quem mensure apenas três regiões, axilar, xifoide e basal [22,23]. Quanto aos valores considerados normais também não há um consenso, podendo variar de 6 a 7 [20,22], 4 a 7 [23] e até de 5 a 11 [24], sendo que medidas entre 3 a 4 centímetros corresponderiam a uma capacidade pulmonar $20 \%$ abaixo do normal [22].

Os resultados do presente estudo mostraram que as medidas da mobilidade torácica, antes e após a intervenção por um programa de EFS, apresentaram, em todas as quatro regiões mensuradas, médias abaixo de 3,5 cm, ou seja, segundo Carvalho [22], as participantes avaliadas possuíam capacidade pulmonar abaixo do normal. Este achado pode ser explicado pelo processo de envelhecimento que predispõe à diminuição da mobilidade torácica, quando comparada à de indivíduos jovens [25]. Além disso, o idoso utiliza quase duas vezes mais do que um jovem a elasticidade total de sua parede torácica. A redução na elasticidade e a hipotrofia dos músculos respiratórios reduz a capacidade de expansibilidade da caixa torácica e incrementa o volume residual [26-28].

Ao comparar as avaliações inicial e final do programa de EFS, observaram-se ganhos significativos nas médias da expansibilidade da região axilar no P3 e na das $12^{\text {as }}$ costelas no $\mathrm{P} 2$, que podem ser atribuídos à realização de alongamentos musculares e à mobilização de articulações envolvidas na mecânica respiratória, além da realização de manobras respiratórias lentas e profundas, que aconteceram principalmente durante a execução dos exercícios de conscientização corporal e relaxamento. Estudos envolvendo adultos jovens submetidos a um programa de alongamento da musculatura respiratória e de reeducação postural global (RPG) [29] e indivíduos portadores de doença pulmonar obstrutiva crônica submetidos a um programa educacional e a um programa de exercícios físicos objetivando o aumento da mobilidade torácica [30], também obtiveram resultados positivos quanto ao aumento da mobilidade torácica, corroborando, desta forma, com o presente estudo.

O aumento da idade conduz a uma diminuição das medidas de capacidade pulmonar, o que tem relação com a expansibilidade torácica [31]. Porém, quando realizamos a comparação intergrupos ( 2 2 vs. P3), não observamos diferença em nenhum dos CR (axilar, xifoide, $12^{\text {as }}$ costelas ou umbilical) tanto antes quanto após o programa de EFS, sugerindo assim que a diferença na média de idade de quase uma década a mais para $\mathrm{P} 2$ e a realização de um dia a mais por semana de EFS para P3 não foram capazes de promover diferenças significativas na mobilidade torácica.

A obesidade é outro fator que influencia bastante a complacência pulmonar, levando à diminuição da mobilidade da parede torácica, efeito justificado pela grande deposição de tecido adiposo no tórax e no abdome, determinando o aumento da retração elástica e redução da distensibilidade das estruturas extrapulmonares, mesmo havendo normalidade do sistema respiratório [32,33]. Contudo, a obesidade não foi uma variável interferencial quando comparamos P2 e P3, visto que ambos os grupos apresentavam-se classificados como sobrepeso segundo a Organização Mundial da Saúde [34]. Entretanto, o sobrepeso pode ter influenciado, juntamente com o envelhecimento, nas baixas médias iniciais e finais de ambos os grupos.

Apesar do IMC ser uma medida bastante utilizada, não deve ser o único parâmetro para a estimativa de obesidade ou massa corporal gorda, principalmente em idosos, visto que não distingue adequadamente massa gorda e MM. Indivíduos de idade mais avançada possuem maior quantidade de GC em um determinado IMC quando comparados a indivíduos jovens; por este motivo, alguns autores consideraram que o IMC possa ser um indicador menos útil de adiposidade $[35,36]$. Assim sendo, este estudo utilizou o método da bioimpedância para uma análise mais fidedigna da composição corporal das idosas. Apesar de o IMC ter permanecido praticamente o mesmo antes e após o EFS, tanto em P2 quanto em P3, no P2 foram observados aumento da MM e declínio da GC após a prática de EFS, enquanto no $\mathrm{P} 3$ não houve alteração em nenhuma dessas variáveis. A menor média da GC no P2 em relação ao P3, tanto antes como depois do programa, pode estar relacionada ao aumento da gordura nas primeiras décadas do envelhecimento, bem como à perda de gordura nas décadas mais tardias, padrão que parece ser o mais provável no comportamento da adiposidade corporal durante o processo de envelhecimento [37,38]. Já em relação à restrição/acompanhamento nutricional nada pode ser referido, pois não houve diferença significativa entre a percentagem de participantes que os realizaram.

$\mathrm{O}$ ganho significativo de MM no P2 pode estar relacionado ao treinamento de força muscular durante 
o EFS. Esse fator pode se justificar pelo retardamento do processo de sarcopenia com o uso de sobrecargas, obtendo expressivas respostas neuromusculares (hipertrofia e força muscular) e por aumento da capacidade contrátil dos músculos esqueléticos [39]. Assim sendo, indaga-se sobre quais os motivos de P3 não apresentar os mesmos resultados quanto à $\mathrm{MM} \mathrm{em}$ relação a $\mathrm{P} 2$, visto que ambos os grupos realizaram o mesmo tipo de EFS, sendo maior o número de dias por semana no P3. Considerando esta indagação, deve-se levar em conta que modificações na GC e na MM não podem ser padronizadas durante o envelhecimento, pois outros aspectos do estilo de vida, como por exemplo, atividade física aumentada, podem alterar algumas dessas variáveis de composição corporal, resultando em desaceleração no aumento da adiposidade relacionada à idade ou mesmo no seu declínio, diminuição da MM ou até mesmo seu ganho [40]. Podemos atribuir a ausência de mudanças na composição corporal em P3 ao fato de apenas $10 \%$ desse grupo, contra quase metade de $\mathrm{P} 2$, terem realizado outros tipos de exercício físico durante o período do programa de EFS. Isso demonstra que qualquer tipo de exercício físico pode ser favorável para as pessoas de terceira idade. É possível que o programa de EFS desenvolvido duas vezes por semana tenha estimulado as idosas a se tornarem mais ativas, dessa forma transcendendo os efeitos dos exercícios realizados durante as sessões do programa, para uma mudança benéfica no estilo de vida. Uma limitação deste estudo é que não foi possível concluir se o número de sessões realizadas pelos grupos foi um fator determinante dos efeitos encontrados, visto que diferenças existentes entre os dois grupos, como idade e realização de exercícios além do programa de EFS, podem ter influenciado os resultados encontrados.

Apesar dessas limitações, estes resultados sugerem que, apesar do processo gradual de envelhecimento ser intensificado durante a terceira idade devido às grandes modificações no organismo do idoso, mulheres idosas demonstraram alguma melhora na expansibilidade toracoabdominal, aumento da massa magra e diminuição e/ou manutenção da gordura corporal, após seis meses de um programa de EFS com sessões duas ou três vezes por semana. Esse pequeno ganho para a população idosa pode levar a relevantes melhoras, tanto nos aspectos biológicos quanto nos psíquicos e sociais.

\section{REFERÊNCIAS}

1. Rebelatto JR, Calvo JI, Orejuela JR, Portillo JC. Influência de um programa de atividade física de longa duração sobre a força muscular manual e a flexibilidade corporal de mulheres idosas. Rev Bras Fisioter. 2006;10(1):127-32. http://dx.doi.org/10.1590/S1413-35552006000100017

2. Rosset I, Cruz MR, Santos JLF, Haas VJ, Wehbe SCCF, Rodrigues RAP. Diferenciais socioeconômicos e de saúde entre duas comunidades de idosos longevos. Rev Saude Publica. 2011;45(2):391-400. http://dx.doi.org/10.1590/S0034-89102011000200018

3. Civinski C, Montibeller A, Braz ALO. A importância do exercício físico no envelhecimento. Rev da Unifebe. 2011;9(1):163-75.

4. Baltes PB. Theoretical Propositions of Life-Span Developmental Psychology: On the Dynamics Between Growth and Decline. Developmental Psychology. 1987;23(5):611-26. http://dx.doi.org/10.1037/0012-1649.23.5.611

5. Teixeira DC, Prado Junior SRR, Lima DF, Gomes SC, Brunetto AF. Efeitos de um programa de exercício físico para idosas sobre variáveis neuro-motoras, antropométricas e medo de cair. Rev Bras Educ Fís Esp. 2007;21(2):107-20.

6. Mazo GZ, Külkamp W, Lyra VB, Prado APM. Aptidão funcional geral e índice de massa corporal de idosas praticantes de atividade física. Rev Bras Cineantropom Desempenho Hum. 2006;8(4):46-51.

7. Matsudo SM, Matsudo VR, Araújo T, Andrade D, Andrade E, Oliveira L, Braggion G. Nível de atividade física da população do Estado de São Paulo: análise de acordo com o gênero, idade, nível sócio-econômico, distribuição geográfica e de conhecimento. Rev Bras Ci Mov. 2002;10(4):41-50.

8. Mazo GZ, Mota J, Gonçalves LHT, Matos MG. Nível de atividade física, condições de saúde e características sócio-demográficas de mulheres idosas brasileiras. Rev Port Cien Desp. 2005;2:202-12.

9. Gome KV, Zazá DC. Motivos de adesão a prática de atividade física em idosas. Rev Bras de Ativ Física \& Saúde. 2009;14(2):132-8.

10. Jacob Filho W. Atividade física e envelhecimento saudável. Rev Bras Educ Fís Esp. 2006;20(5):73-5.

11. Forbes G. Body composition: influence of nutrition, physical activity, growth and aging. In: Forbes G. Modern nutrition in health and disease. Baltimore: Williams \& Wilkins; 1999.

12. Kushner RF. Bioelectrical impedance analysis: a review of principles and applications. J Am Coll Nutr. 1992;11(2):199-209.

13. Chumlea WC, Guo SS, Kuczmarski RJ, Vellas B. Bioelectrical and anthropometric assessments and reference data in the elderly. J Nutr. 1993;123:449-53.

14. Belini M. Força muscular respiratória em idosos submetidos a um protocolo de cinesioterapia respiratória em imersão e em terra [Monograph]. [Panamá]: Centro de Ciências Biológicas e da Saúde da Universidade; 2004. 
15. Rasslan Z, Junior RS, Stirbulov R, Fabbri RMA,Lima CAC. Evaluation of pulmonary function in class I and II obesity. J Bras Pneumol. 2004;30(6):508-14. http://dx.doi.org/10.1590/S1806-37132004000600004

16. Goya W, Gerald S, Peter HW. Body fat distribuation, body composition and respiratory function in elderly men. Am J Clin Nutr. 2005;82:996-1003.

17. Souza JN, Chaves EC. O efeito do exercício de estimulação da memória em idosos saudáveis. Revista da Escola de Enfermagem da USP. 2005;39(1):13-9. http://dx.doi.org/10.1590/S0080-62342005000100002

18. Lehmkuhl, E, Neves FM, Panizzi EA, Pamplona CMA, Kerkoski E. A mobilidade torácica avaliada em diferentes regiões através da técnica de cirtometria em indivíduos saudáveis. Anais do IX Encontro Latino Americano de Iniciação Científica e V Encontro Latino Americano de Pós-Graduação da Universidade do Vale do Paraíba, 2007; São José dos Campos, Brasil.

19. Lukaski HC. Methods for the assessment of human body composition: traditional and new. Am J Clin Nutr. 1987;46:537-56.

20. Azeredo CAC. Fisioterapia respiratória. São Paulo: Manole; 1984.

21. Costa D. Fisioterapia respiratória básica. São Paulo: Atheneu; 1999.

22. Carvalho A. Semiologia em reabilitação. São Paulo: Atheneu; 1994.

23. Lianza S. Medicina de reabilitação. 2aㅡ ed. Rio de Janeiro: Guanabara Koogan; 1995.

24. Bethlem N. Pneumologia. 4를. São Paulo: Atheneu; 1995.

25. Carmano FA; Durigon OFS, Landaburu C, Pardo MS. Estudo comparativo de duas técnicas de avaliação da mobilidade torácica em mulheres jovens e idosas saudáveis. Fisioterapia Brasil. 2003;4(5):348-52.

26. Turner JM, Mead J, Wohl ME. Elasticity of human lungs in relation to age. J Appl Physiol. 1968;25:664-71.

27. Grimby G. Physical activity and effects of muscle training in the elderly. Ann Clinic Res. 1988;20:62-6.

28. Papaléo MN. Gerontologia: a velhice e o envelhecimento em visão globalizada. São Paulo: Atheneu; 2002.

29. Teodori RM, Moreno MA, Piore Junior JF, Oliveira ACS. Alongamento da musculatura inspiratória por intermédio da reeducação postural global (RPG). Rev Bras Fisioter. 2003;7(1)25-30.

30. Paulin E, Brunetto AF, Carvalho CRF. Efeitos de programa de exercícios físicos direcionado ao aumento da mobilidade torácica em pacientes portadores de doença pulmonar obstrutiva crônica. J Pneumologia. 2003;29(5):287-94. http://dx.doi.org/10.1590/S010235862003000500007

31. Ruivo S, Viana P, Martins C, Baeta C. Efeito do envelhecimento cronológico na função pulmonar. Comparação da função respiratória entre adultos e idosos saudáveis. Rev Port Pneumol. 2009;4(4):629-53. http://dx.doi.org/10.1016/S0873-2159(15)30161-6

32. Naimark A, Cherniack RM. Compliance of the respiratory system and its components health and obesity. J Appl Physiol. 1960;377-82.

33. Wadström C, Muller-Suur R, Backman L. Influence of Excessive Weight Loss on Respiratory Function. Eur J Surg. 1991;157(5):341-6.

34. World Health Organization. Expert Committee: physical status: the use and interpretation of anthropometry. Geneva: World Health Organization; 1995.

35. Landi F, Onder G, Gambassi G, Pedone C, Carbonin P, Bernabei R. Body mass index and mortality among hospitalized patients. Arch Intern Med. 2000;160(17):2641-4. http://dx.doi.org/10.1001/archinte.160.17.2641

36. Cervi A, Franceschini SCC, Priore SE. Análise crítica do uso do índice de massa corporal para idosos. Rev Nutr. 2005;18(6)765-75. http:// dx.doi.org/10.1590/S1415-52732005000600007

37. Going S, Williams D, Lohman T. Aging and body composition: biological changes and methodological issues. In: Hollozy JO (Ed.). Exer Sport Sci Reviews. 1995;23:411-49. http://dx.doi.org/10.1249/00003677-199500230-00016

38. Fiatarone-Singh M A. Body composition and weight control in older adults. In: Lamb DR, Murray R. Perspectives in exercise science and sports medicine: exercise, nutrition and weight control. Carmel: Cooper Publishing; 1998. p 243-88.

39. Silva CM, Gurjão ALD, Ferreira L, Gobbi LTB, Gobbi S. Efeito do treinamento com pesos, prescrito por zonas de repetições máximas, na força muscular e composição corporal em idosas. Rev Bras Cineantropom Desempenho Hum. 2006;8(4):39-45.

40. Kyle UG, Genton L, Gremion G, Slosman DO, Pichard C. Aging, physical activity and height-normalized body composition parameters. Clin Nutr. 2004;23(1):79-88. http://dx.doi.org/10.1016/S0261-5614(03)00092-X C 